\title{
A CHARACTERISTIC EXTREMAL PROPERTY OF SIMPLICES
}

\author{
ROLF SCHNEIDER
}

\begin{abstract}
Among all $d$-dimensional convex bodies the simplices are characterized by the property that the volume of every circumscribed cylinder is not less than $d$ times the volume of the body itself.
\end{abstract}

For every convex body $K$ with interior points in $d$-dimensional Euclidean space $E^{d}$ there exists a cylinder $Z$ with the properties $K \subseteq Z$ and $V(Z) \leqq$ $d V(K)$ (as follows from Lemma 6 of Macbeath [6]). Here $V$ denotes the volume, and by a cylinder in $E^{d}$ we understand any $d$-dimensional convex body which is the Minkowski sum of a $(d-1)$-dimensional convex body and a line segment. Butler [3] has shown that $V(Z) \geqq d V(K)$ for every simplex $K$ in $E^{d}$ and all cylinders $Z$ circumscribed about $K$. Hence the functional $f$ (a "measure of noncylindricity", as it might be called) defined by

$$
f(K)=\inf \{V(Z) / V(K): K \subseteq Z \text { and } Z \text { a cylinder }\}
$$

for every $d$-dimensional convex body $K$, attains its maximum if $K$ is a simplex. Butler [3, Remark 3, p. 560] left open the question whether simplices are the only extremal bodies. This question is answered affirmatively by the following result.

Suppose $K \subset E^{d}$ is a d-dimensional convex body which satisfies $V(Z) \geqq$ $d V(K)$ for every circumscribed cylinder $Z$. Then $K$ is a simplex.

Proof. We denote the convex hull of a set $M$ by conv $M$ and its affine hull by aff $M$. Suppose $K$ is a $d$-dimensional convex body in $E^{d}$. Choose a regular boundary point $p$ of $K$. Through $p$ there exists a unique supporting hyperplane $H$ of $K$. Let $H^{*}$ be the other supporting hyperplane of $K$ parallel to $H$. Choose a point $q \in K \cap H^{*}$ and let $C$ be the infinite convex cylinder consisting of all lines parallel to $L_{0}=\operatorname{aff}\{p, q\}$ which meet the body $K$. Write $B=C \cap H$ and $P=\operatorname{conv}(B \cup\{q\})$. If $L_{1}$ is a line parallel to $L_{0}$ and different from $L_{0}$ which meets the interior of $K$, then the set of real numbers $\lambda$ for which the line $L_{\lambda}=(1-\lambda) L_{0}+\lambda L_{1}$ meets $K$ contains a

Received by the editors October 19, 1972 and, in revised form, December 21, 1972. AMS (MOS) subject classifications (1970). Primary 52A20, 52A40.

Key words and phrases. Convex body, simplex, circumscribed cylinder, volume.

(c) American Mathematical Society 1973 
greatest element $\Lambda$. If $b$ is the intersection point of $L_{\Lambda}$ and $H$, then

$$
L_{1} \cap P=L_{1} \cap \operatorname{conv}\{p, q, b\} .
$$

If this were false, there existed a point $x \in L_{1} \cap P$ such that $x \notin L_{1} \cap$ $\operatorname{conv}\{p, q, b\}$. The point $x$ is contained in the convex hull of $q$ and some point $y \in B$. The line through $y$ parallel to $L_{0}$ meets $K$. But this contradicts the maximality of $L_{\Lambda}$, since clearly the segment $\operatorname{conv}\{p, y\}$ contains the point $b$ in its relative interior.

Choose a point $a \in L_{\Lambda} \cap K$. As

$$
L_{1} \cap K \supseteq L_{1} \cap \operatorname{conv}\{p, q, a\}
$$

and the segments on the right-hand sides of (1) and (2) are equal in length, it follows that $L_{1} \cap P$ is not longer than $L_{1} \cap K$. Since $L_{1}$ may be chosen arbitrarily in $C$ we deduce

$$
V(P) \leqq V(K) .
$$

Let $Z$ denote the compact cylinder which is cut out from $C$ by the hyperplanes $H$ and $H^{*}$. From $V(Z)=d V(P)$ we deduce $V(Z) \leqq d V(K)$.

Now suppose that $K$ satisfies $V(Z) \geqq d V(K)$ for every circumscribed cylinder $Z$. Then equality must hold in (3). This implies

$$
L_{1} \cap K=L_{1} \cap \operatorname{conv}\{p, q, a\},
$$

hence the segment $\operatorname{conv}\{p, a\}$ contains a boundary point $c$ of $K$ in its relative interior. Then each supporting hyperplane of $K$ through $c$ contains $p$ and therefore coincides with $H$. This shows $a \in H$, hence $a=b$. From (1), (4) and the arbitrariness of $L_{1}$ we deduce $K=P$. Since $p$ was an arbitrary regular boundary point of $K$, we have shown that $K$ has the following property:

(5) For every regular boundary point $p$ of $K$ there exist a supporting hyperplane $H$ of $K$ and a point $q$ of $K$ such that $p \in H$ and

$$
K=\operatorname{conv}((H \cap K) \cup\{q\}) .
$$

Let $H$ and $q$ be as in (5). As $K$ has interior points, the face $H \cap K$ is $(d-1)$-dimensional. Choose a point $p^{\prime}$ which is a regular boundary point of $H \cap K$ if this set is considered as a full-dimensional convex set in $H$. Then every point $r$ in the relative interior of the segment $\operatorname{conv}\left\{p^{\prime}, q\right\}$ is a regular boundary point of $K$. By (5) there exist a supporting hyperplane $J$ of $K$ and a point $q^{\prime}$ of $K$ with the properties $r \in J$ (which implies $p^{\prime} \in J$ ) and $K=\operatorname{conv}\left((J \cap K) \cup\left\{q^{\prime}\right\}\right)$. Since $q^{\prime}$ is contained in the convex hull of $q$ and some point $s$ of $H$, and $s$ is contained in the convex hull of $q^{\prime}$ and 
some point of $J$, we have $q^{\prime}=s \in H$. We deduce

$$
H \cap K=H \cap \operatorname{conv}\left((J \cap K) \cup\left\{q^{\prime}\right\}\right)=\operatorname{conv}\left(\left(H^{\prime} \cap H \cap K\right) \cup\left\{q^{\prime}\right\}\right),
$$

where $H^{\prime}=J \cap H$ is a $(d-2)$-dimensional flat in $H$ which supports $H \cap K$ and contains $p^{\prime}$. We have proved that $H \cap K$, considered as a body in $(d-1)$-dimensional space, has property (5). Now we can use induction with respect to the dimension to show that a $d$-dimensional convex body satisfying (5) is a simplex; the case $d=2$ is, in fact, trivial.

REMARKS. For $d=2$ our result is contained implicitly in investigations of Estermann [4, p. 472] and Levi [5, p. 126].

It should be mentioned that our method of proof is related to the process of "shakedown" ("Schüttelung"), which has proved useful in establishing other extremal properties of simplices; see, e.g., Blaschke [1, p. 58], Bonnesen-Fenchel [2, p. 71].

\section{REFERENCES}

1. W. Blaschke, Vorlesungen über Differentialgeometrie, vol. II, Springer, Berlin, 1923.

2. T. Bonnesen and W. Fenchel, Theorie der konvexen Körper, Springer, Berlin, 1934.

3. G. J. Butler, An extremal property of simplices, Proc. Amer. Math. Soc. 30 (1971), 556-560. MR 44 \#2140.

4. T. Estermann, Über den Vektorenbereich eines konvexen Körpers, Math. Z. 28 (1928), 471-475.

5. F. W. Levi, Über zwei Sätze von Herrn Besicovitch, Arch. Math. 3 (1952), 125-129. MR 14, 309.

6. A. M. Macbeath. A compactness theorem for affine equivalence-classes of convex regions, Canad. J. Math. 3 (1951), 54-61. MR 13, 577.

Fachbereich Mathematik, Technische Universität Berlin, Berlin, Germany 